\title{
Quantitative Assessment of Colorectal Cancer Progression: a Comparative Study of Linear and Nonlinear Microscopy Techniques
}

BACKGROUND AND AIMS: Colorectal cancer (CRC) is a disease that can be prevented if is diagnosed and treated at pre-invasive stages. Thus, the monitoring of colonic cancer progression can improve the early diagnosis and detection of malignant lesions in the colon. This monitoring should be performed with appropriate image techniques and be accompanied by proper quantification to minimize subjectivity. We have monitored the mice CRC progression by image deconvolution, two-photon emission fluorescence (TPEF) and second harmonic generation (SHG) microscopies and present different quantization indices for diagnosis. METHODS: The Azoxymethane (AOM) / dextran sodium sulfate (DSS) protocol was used. 35 eight-week old male BALB/cCmedc mice were used and distal colon segments were dissected at day zero and fourth, eighth, sixteen, and twenty weeks after injection. These segments were observed with linear and nonlinear optical microscopies and several parameters were used for quantification. RESULTS: Crypt diameter higher than $0.08 \mathrm{~mm}$ and increased fluorescence signal intensity in linear images; as well as aspect relation above 0.7 and altered organization reflexed by high-energy values obtained from SHG images, away from those obtained in normal tissues. CONCLUSION: the combination of linear and nonlinear signals improve the detection and classification of pathological changes in crypt morphology/distribution and collagen fiber structure/arrangement. In combination with standard screening approaches for CRC, the proposed methods improve the detection of the disease in its early stages, thereby increasing the chances of successful treatment. KEYBORDS: colorectal cancer, linear microscopy, nonlinear microscopy, early diagnosis 


\section{INTRODUCTION}

34 Colorectal cancer ( $\mathrm{CRC})$ is a common malignancy, it is the third most common cause of cancer death in both men and women [1]. In general, CRC has a good prognosis, when it is diagnosed and treated at pre-invasive stages [2]. However, the detection of early lesions is still challenging.

To systematically characterize early tumoral transformations, an animal model of CRC progression is needed. Mice Azoxymethane (AOM)-induced CRC is one of the most commonly used pre-clinical animal model to mimic human sporadic CRC development [3]. Specifically, the AOM/dextran sodium sulfate (DSS) protocol has been proven a powerful tool for investigating the pathogenesis and chemoprevention of colitis-related colon carcinogenesis [4]. The development of colonic cancer is a multi-stage process [5] and the monitoring of colonic cancer progression can improve the early diagnosis and detection of malignant colon lesions. This examining should be performed with appropriate image techniques and be accompanied by proper quantification to minimize subjectivity.

Currently, anatomo-pathological examination is the diagnostic gold standard. The process involves many steps, prior to examination. It is time-consuming and expensive, and the excision itself has the inherent risks of bleeding and perforation. Thus, new imaging technologies might be tremendously helpful. Many examination techniques, such as computed tomography (CT), positron emission tomography (PET), magnetic resonance imaging (MRI), endorectal ultrasound (ERUS) lack the needed resolution or require exogenous contrasting agents [6]. Confocal endomicroscopy can reveal histological details during ongoing endoscopy. However, the usefulness of this imaging modality in patients is limited because of the need of fluorescent dyes [7]. Nonlinear and linear microscopy techniques provide unprecedented information that might help to overcome these limitations. 
57 Two-photon excitation fluorescence (TPEF), second harmonic generation (SHG), third

58 harmonic generation (THG), and coherent anti-Stokes Raman scattering (CARS) microscopy

59 exhibit several diagnostic advantages. These techniques are label-free, offer inherent three-

60 dimensional resolution, use near-IR excitation resulting in superior optical penetration, low

61 photodamage, and provide quantitative information [8]. In the last years, the nonlinear

62 techniques have proven to be efficient for the diagnosis of CRC [9, 10]. Similarly, confocal

63 or deconvolution microscopy, with optical sectioning capacity and exploring tissue

64 autofluorescence (i.e. label free) were also used for quantitative and diagnostic purposes [11, 65 12].

66 Contrast mechanisms in conventional optical imaging are based on absorption, reflection,

67 scattering and fluorescence, and the response recorded is linearly dependent on the intensity

68 of the incident light (mercury lamp or continuous wave [CW] laser). In contrast, nonlinear

69 optical effects used as the imaging contrast, occur when a biological tissue interacts with an

70 intense laser beam (pulsed laser) and exhibits a nonlinear response to the applied field

71 strength [13] (Fig. 1). Linear technique is cheaper and easily to operate while nonlinear needs

72 specific and expensive instrumentation and qualified operator. Nonlinear optic presents

73 better tissue penetration due to reduced scattering of larger wavelengths. However, both tools

74 could be useful for the monitoring of CRC progression.

75

76

77

Fig 1: Schematic representation and Jablonski diagram of linear (one-photon emission fluorescence) and nonlinear (two-photon emission fluorescence and second harmonic generation) microscopy techniques (left column). Representative free-label (not stained) images of the mouse colon mucosa obtained respectively with these microscopy techniques are shown (right column). 
81 In this work, we have examined CRC progression by image deconvolution, TPEF and SHG

82 microscopies and present a detailed evaluation of the tumoral transforming colonic mucosa.

83 We introduce the principle of operation and properties of each microscopy and present

84 different quantization indices for diagnosis, discussing in depth the application of these novel

85 techniques in a mouse model of CRC.

86

87 MATERIALS AND METHODS

88 - Murine model of AOM/DSS-induced colon cancer

89 The study was previously approved by the Ethics Committee for Animal Research of the

90 National University of Entre Ríos. The use of laboratory animals followed the ethical code

91 of International Organization of Medical Sciences for animal's experimentation. The experimental population consisted of 35 eight-week old male BALB/cCmedc mice weighing 20g-30g. After 7 days of acclimatization, animals were randomized into experimental

94 (AOM/DSS-treated) and control (saline-injected) groups. AOM/DSS-treated animals were

95 intraperitoneally injected with azoxymethane (AOM) $0.01 \mathrm{mg} / \mathrm{g}$ body weight. One week 96 later, they were given dextran sodium sulphate (DSS) in the drinking water for seven days, 97 according to Tanaka and co-workers [14]. Distal colon segments were dissected at day zero 98 and fourth, eighth, sixteen, and twenty weeks after injection (AOM-injected mice were not evaluated at the week 0) (Fig. 2A). These segments were destined to histological processing 100 (for control purposes, Fig. 2B) and linear (Fig. 2C) and nonlinear optical (Fig. 2D) microscopy observation (for quantification).

Fig 2: Experimental setup of mouse-CRC chemical induction. A) Mice were injected with AOM or saline solution (control) at day 0, one week later, DSS was supplied in drink water during seven days. Next animals 
105

106

107

108

109

110

111

112

113

114

115

116

117

118

119

120

121

122

123

were sacrificed at weeks 4th, 8th, 16th, and 20th (W4, W8, W16, W20). Top row pictures show the representative appearance of the anal region of control and AOM/DSS treated mice. Below is the row in which distal colon segments of control and CRC progression stages are shown. B) Representative H\&E-stain sections used as gold standard. a: shortened crypts, b: bifurcated crypts, c: fused crypts, d: severe dysplasia, e: goblet cell depletion, f: lymphocytic infiltrate, g: dirty necrosis zone, h: absence of goblet cells, i: severe dysplasia, j: lymphocytic infiltrate. C) Representative images obtained with linear microscopy, and D) Representative nonlinear microscopy: green (TPEF) and red (SHG) signals, respectively.

\section{- Linear Microscopy}

A fluorescence deconvolution microscopy system was used as linear setup. This technique included: (a) optical-sectioning of the autofluorescent distal colon segments (producing a 3D image stacks), (b) Point Spread Function (PSF) determination, (c) deconvolution of the 3D image stacks, (d) 2D or 3D quantifications. The system used was based on an Olympus BX50 upright microscope; a complete description of the system has been previously published [15] (Fig. S1A, supplementary material). Deconvolution was performed using the DeconvolutionLab plugin from ImageJ [16]. Colon mucosa auto-fluorescence was observed at different wavelengths (Fig. 2C). Table 1 lists the major components identified by the autofluorescence.

Table 1: The origins of principal intrinsic signals in colon mouse

\begin{tabular}{|c|l|l|l|}
\cline { 2 - 4 } \multicolumn{1}{c|}{} & \multicolumn{1}{c|}{ Linear Microscopy } & \multicolumn{2}{c|}{ Nonlinear Microscopy } \\
\hline \hline \multirow{4}{*}{ Layer } & Autofluorescence & \multicolumn{1}{|c|}{ TPEF } & \multicolumn{1}{c|}{ SHG } \\
\hline & FAD & NADH & Collagen fibers \\
& Porphyrin derivatives & FAD & \\
& Lipopigments & Elastic fibers & \\
& & Eosinophils & \\
& & Lipopigments & \\
\hline \multirow{2}{*}{ Submucosa } & & Elastic fibers & Collagen fibers \\
& & Collagen bundles & Collagen bundles \\
\hline
\end{tabular}


125 - Nonlinear Microscopy

126 The system consisted of an inverted microscope Axio Observer Z.1 equipped with a Zeiss

127 LSM 780-NLO confocal scan head (Carl Zeiss AG, Okerkochen, Germany). The excitation

128 beam was provided by a mode-locked Ti:Sapphire laser (Spectra-Physics. Irvine, CA, USA)

129 at the wavelength of $940 \mathrm{~nm}$ and an average power at the sample of $10 \mathrm{~mW}$. The forward

130 propagating SHG $(\lambda=470 \mathrm{~nm})$ and two-photon fluorescence signals $(\lambda>490 \mathrm{~nm})$ were

131 acquired simultaneously by a non-descanned detector (NDD). A complete description of the

132 system has been published elsewhere [17]. Figure 2D shows representative images acquired

133 with this configuration; major components identified in this system are listed in Table 1 . The

134 microscope structure can be seen in Figure S1B (Supplementary material).

135 - Acquisition Strategy

136 Murine colon segments were longitudinally splitted and fresh mucosa optically sectioned in 137 apical-basal direction to acquire linear autofluorescence images (Fig. 3). Optical sectioning 138 was performed to obtain tissue information through the crypt axis, up to $40 \mu \mathrm{m}$ depth. After 139 acquisition and before quantification, all images were deconvolved. Distal colon serial 140 segments of each animal, in turn, were fixed, embedded on paraffin, sectioned to $5 \mu \mathrm{m}$

141 thickness and stained with hematoxilin and eosin (HE) to classical histological analysis 142 (methodological control). In addition, some sections were stained with the Schmorl and 143 Fouchet stain [18] to detect lipofuscins and porphyrins. On the other hand, cross-sectioned 144 murine colon segments were freshly observed to obtain nonlinear TPEF and SHG images 145 (Fig. 3). Distal colon serial segments of each animal, in turn, were processed to histological 146 analysis by HE-stain. 
147 Fig 3: General methodological strategy: Top panel shows images acquisition from colon longitudinal sections.

148 The open fresh mucosa is optically sectioned, and then deconvolved to obtain images at different depths; blue

149 square: surface (depth 0), red square: superficial epithelium (5-10 $\mu \mathrm{m}$ depth) and yellow square: deep mucosa

150 (30-40 $\mu \mathrm{m}$ depth). Bottom panel shows different representation possibilities of nonlinear TPEF (green) and

151 SHG (red) images obtained from fresh tissue cross sections.

153 Three animals (saline-injected) were euthanized at time 0 and used as controls (time/week

154 0). The remaining 32 were euthanized at four, eight, sixteen and twenty weeks after the

155 chemical injection saline or AOM. For each experimental point of AOM/DSS-treated 156 animals, 8 mice were injected: 5 with AOM (colon cancer model) and 3 saline solution

157 (control). For each colon segment, images from three different zones in the plane were 158 captured in 2D studies and one sectioning of $40 \mu \mathrm{m}$ (linear) or $100 \mu \mathrm{m}$ (nonlinear) thickness

159 for 3D analysis.

160 - Quantification methodologies

161 The analysis of colon cancer progression was evaluated both at morphological and 162 biochemical level by colon deconvolved autofluorescence images. For the morphological 163 analysis was applied the Kudo's pit pattern classification, to determine manual- and 164 automatically, the shape and pit pattern of the colon crypt pits. Briefly, superficial colon 165 crypts were categorized using Kudo's classification, according to appearance, shape and 166 perimeter [19]. For automatic analysis, 2D deconvolved images were segmented to identify 167 crypt morphology. While Types I and II were considered benign changes, the pit pattern 168 classes III-V were classified as neoplastic and malignant changes.

169 The biochemical analysis of the endogenous fluorophores changes was done for intensity 170 measurements. Briefly, 2D images were opened in ImageJ. To avoid out-of-focus or noisy 
171 areas, a square template of $8 \times 8$ ROI (region of interest), each one of $64 \times 64$ pixels, was drawn

172 and positioned on the image. Twenty ROIs were selected from the template to quantify the

173 intensity. Using the multi-measure plugin, integrated density was measured for each ROI.

174 Finally, considering a linear behavior of the CCD camera and the independence of the

175 exposure time $\left(\mathrm{t}_{\mathrm{exp}}\right)$, the following formula was used to obtain the average gray level value

176 (I): $\left[\mathrm{I}=\left(\mathrm{I}_{\text {average }}-\mathrm{I}_{\text {background }}\right) / \mathrm{t}_{\text {exp }}\right]$, where $\mathrm{I}_{\text {average }}$ is the integrated intensity measure, $\mathrm{I}_{\text {background }}$

177 correspond to intensity level register without sample, and $t_{\exp }$ is the capture time.

178 In parallel, a morphological analysis of the colon cancer progression also was done from

179 nonlinear TPEF and SHG images. The methods of nonlinear image analysis were carried out

180 with ImageJ software and calculations were made for 16 ROIs (256 x 256 pixels) chosen

181 over selected images. For SHG intensity amount, signal was separated from the background

182 with a threshold at level 50 from the 0 to 255 gray levels. From each ROI the area fraction

183 (essentially a measure of SHG prevalence) was quantified. To achieve the aspect ratio (AR),

184 Fast Fourier Transform (FFT) was used [20]. If the fibers have a parallel arrangement, the

185 intensity plot of the FFT image, look as an ellipse and consequently will have a higher AR.

186 In contrast, fiber with aleatory arrangement, exhibit an intensity plot of the FFT image, closer

187 to the shape of a circle and with low AR. Texture features analysis, on the other hand, was

188 based on the calculation of correlation (C) and energy (E) of the gray level co-occurrence

189 matrix (GLCM) of the images [21]. The texture analyses were performed by GLCM-Texture

190 plugin from Image-J, which was described by Walker and collaborators [22].

191 - Statistics

192 For multi-group comparisons, one-way analysis of variance (ANOVA) and a post-hoc

193 Tukey-Kramer test were used. In addition, t-testing was applied for two-group comparisons.

194 The level of significance employed was significant $(*) p<0.05$ and very significant $(* *)$ 
195

196 software.

197

198

199

200

201

202

203

204

205

206

207

208

209

210

211

212 circular crypt lumens were observed (Fig. S2E).

213

214 The changes on quantities and subtypes of tissular fluorophores make possible evaluate the

215 development and progression of tumoral processes. Thus using excitation wavelengths

216 between 460 and $550 \mathrm{~nm}$, was possible to register mainly autofluorescence from flavins,

217 porphyrins and lipopigments (Fig. S3, Supplementary material). This endogenous 
218 biomarkers were used to analyze crypts morphology and tissue function changes on the

219 disease progression (Fig. 4, Fig. 6, and Fig. S4).

220 Figure 4 shows the results obtained by processing autofluorescence images. Figure 4A

221 depicts images used for the Kudo's pit pattern classification performed using manual (red

222 lines) and automatic methodologies (black figures). Perimeters values were measured and

223 the morphologies were identified with a help of a pathologist. A comparison table was made

224 using perimeters values obtained from H\&E-images, manual and automatic quantification

225 data (Fig. S4, Supplementary material). Considering the appearance and perimeter of the

226 crypt pits described by Hurlstone and Brown [23]; the Kudo's pit pattern types were

227 identified in the images used as samples. Through the manual methodology, benign changes

228 such as normal round and star-shape pits (type I and II) were detected in time 0 of control

229 samples (W0) and in all weeks of the treated samples (W4, W8, W16 and W20), while tubular

230 pits (type IIIL) of neoplastic changes were also detected in the $16^{\text {th }}$ and $20^{\text {th }}$ weeks. The

231 automatic methodology allowed to characterize more types of pits within the week analyzed

232 and only $10.5 \%$ of pits were classified as "Possible turning to be Type II or IIIL" or "Possible

233 turning to be IIIL" instead of being labelled with a particular Kudo's pit pattern type. In

234 control samples (with physiological solution), the $65 \%$ of the 276 quantified pits were

235 identified as type I, whereas in treated samples, around $50 \%$ of the pits from the $4^{\text {th }}$ and $8^{\text {th }}$

236 pits were type I and the remaining were recognized as neoplastic changes (types III or IV).

237 Data in Figure S4 (Supplementary material) evidence how averaged perimeter values for

238 each period of time remained constant in control samples $(0.06 \mathrm{~mm})$ while they change in

239 equivalent times of treated samples. Finally, the analyses of treated samples must be limited

240 until the $8^{\text {th }}$ week as the pits exhibit a progressive coalescence in the following weeks, which

241 could lead to inaccurate identification and quantification of individual structures. 
242 The analysis of autofluorescence intensity (Fig. 4B) show non-significant changes in the

243 control animals (injected with saline) between weeks $(p>0.05)$. Intensity values range from

2440.9 to 1.6 [arbitrary units, A.U.]. In AOM/DSS-treated animals, whereas the intensity

245 increases slowly at week $4^{\text {th }}(\sim 0.3$ times $)$, it shows a peak $(\sim 3$ times $)$ at week $8^{\text {th }}$ (differences

246 statistically extremely significant $p<0.001)$. Striking, the endogenous fluorophores intensity

247 increases $\sim 1.5$ times at the week $16^{\text {th }}$ and 2.5 times at the week $20^{\text {th }}$. The results found

248 employing two different filters for excitation / emission respectively (460-490 nm / $515 \mathrm{~nm}$;

249 and 480-550 / $590 \mathrm{~nm})$, show similar behavior.

Fig 4: Autofluorescence images quantification. A) Morphological analysis using Kudo classification. First row

252 shows representative images used to perform manual quantification, in which the red color lines (manually

253 drawn), delimit the lumen of the crypts. Second row shows representative images used to perform automatic

254 quantification. After segmentation method, individual crypts (black shapes) are measured. B) Intensity analysis

255 of auto fluorescence images, exemplified in the first figure in which intensity variations (yellow squares) from

256 a square template of $8 \times 8$ ROI, were evaluated. Blue and green bar graph shows intensity for two different filters.

257 P.S: saline IP-injected animals and AOM/DSS: Azoxymethane and Dextran Sodium Sulfate treated animals.

$258 * *$ indicates differences statistically significant $(p<0.05)$ and $* * *$ indicates differences statistically very

259 significant $(p<0.01)$ following ANOVA. All scale bar $=30[\mu \mathrm{m}]$.

262 The combination of TPEF (green) and SHG (red) images enables a superior evaluation of the

263 spatial tissular organization during the disease progression, because the cellular and collagen

264 extracellular matrix compartments configuration, respectively (Figures 5, 6, S5 and S6).

265 Figure S5 (Supplementary material) displays the distribution of collagen fibers in 266 representative mosaic images that allow to observe the complete colon cross-section. 
267 Changes in colon morphology (TPEF images) and collagen distribution (SHG images) were

268 visualized during the disease progression. To confirm this preliminary data, collagen content

269 both in mucosa and submucosa was quantified in each stage of cancer development (Fig. S6,

270 Supplementary material). Collagen content significantly was significantly higher $(p<0.01)$ in

271 the submucosa as compared with mucosa at the different post-injection weeks. Interestingly,

272 collagen content in the submucosa decayed significantly from time 0 (control) (64\%) and

273 week $4^{\text {th }}(16 \%)$, starting to recover from week $8^{\text {th }}(34 \%)$ and staying relatively constant

274 (around 40\%) in weeks $16^{\text {th }}$ and 20 $0^{\text {th }}$ (Fig. S6B). To better understand the behavior of

275 collagen fibers in the submucosa, additional parameters were quantified. Figure 5A shows

276 representative submucosa regions where anisotropy (Fig. 5B), correlation (Fig. 5C) and

277 energy (Fig. 5D) variables were measured. Figure 5B, represents the AR averages, at

278 different disease stages. AR values significantly increase $(p<0.01)$ in diseased samples as

279 compared to the normal colon along time. That means, fibers were arranged in parallel when

280 compared to the AOM/DSS-treated mice samples, i.e. AR showed a more circular

281 configuration (fiber without any specific arrangement). From texture analysis, fibrils

282 correlation fell off sharply with distance at weeks $4^{\text {th }}$ and $8^{\text {th }}$ (Fig. 5C), revealing the existence

283 of isolated thin fibrils. On the other hand, correlation for the fibrils in normal and AOM/DSS-

284 treated mice samples at weeks $16^{\text {th }}$ and $20^{\text {th }}$ remained elevated for larger distances, implying

285 in more connected structures. Consistent with the qualitative observation, evaluation of three

286 ROI (100x100 pixel side squares) in the SHG images showed that the correlation remained

287 higher in the control and weeks $16^{\text {th }}$ and $20^{\text {th }}$; i.e. the Corr50 (the pixel distance where the

288 correlation dropped below $50 \%$ of the initial value) significantly was greater in normal, W16,

289 and W20 as compared with the early stages (W4 and W8) (Fig. 5C; $p<0.05$, ANOVA). Since

290 collagen fiber width and spacing affects the gray levels transition across the image, it is 
291 expected that GLCM energy will change for different collagen morphologies. Fibers in the

292 normal submucosa have lower average energy because they are thinner, causing more

293 variation in gray levels across image (Fig. 5D).

294

295 Fig 5: A) Submucosa TPEF+SHG images during CRC progression. B) Anisotropy calculation using aspect 296 relation (AR). C) and D) texture parameters: correlation (C) and energy (D). Measurements were performed in 2973 ROI of $100 \times 100$ pixel side squared (see white squared). $* * *$ indicates a statistically extremely significant (p $298<0.01)$ difference. Scale bars $=50[\mu \mathrm{m}]$.

301 Figure 6 shows representative 3D images allowing the qualitative analyzes of the auto302 fluorescence and collagen arrangement. Original 3D images of the autofluorescence and 303 deconvolved images (Figure 6A), revealed the morphology of the crypts. TPEF+SHG 3D 304 images (Figure 6B) showed the variation in collagen fiber distribution around the crypts. The quantitative data obtained from the $2 \mathrm{D}$ images are qualitatively identified in the $3 \mathrm{D}$ aspects associated with CRC progression.

Fig 6: Representative 3D visualizations. A) Autofluorescence images before and after deconvolution process.

309 Stack of 40 images, each separate $1 \mu \mathrm{m}$. B) Nonlinear collagen distribution (red). Stack of 100 images, each separate $1 \mu \mathrm{m}$. 


\section{DISCUSSION AND CONCLUSION}

315 Colon cancer is a disease with important repercussions on public health, its diagnosis and the

316 early localization of cell/tissue transformations play an important role in the prevention and

317 curative treatment of colonic cancer. However, physical biopsies do not fully solve this

318 problem because are randomly sampled, and are highly dependent on the skill and experience

319 of physician. One and two photon-induced autofluorescence depends on endogenous

320 fluorophores of biological tissues, which undergo malignant transformation-related changes.

321 Therefore, new techniques for the detection of pathological colonic tissue are needed. This

322 report demonstrates that linear and nonlinear techniques could be used as effective methods

323 for detecting and differentiating the abnormal tissues from the surrounding normal tissues.

324 More importantly, the methods proposed here, not use exogenous markers to detect early

325 changes, making them very promising for early diagnosis.

326 Linear signal was obtained using not-harmful 460 or $550 \mathrm{~nm}$ excitation wavelength range

327 and allowed to precisely identify the lumen crypts of the colon mucosa. Endogenous markers

328 showing fluorescence at these wavelengths were mostly flavins [24, 25], lipopigments, which

329 exhibit bright dotted fluorescence [26, 27], and porphyrins [26-28]. The fluorescence images

330 were used to characterize the crypts morphology and to determine variations in the

331 autofluorescence intensity along the progression of colon cancer. Even though, several

332 groups have reported the utility of autofluorescence to analyze colon tissues [11, 27, 29, 30],

333 according our knowledge, this is the first time that this kind of signal is used to perform Kudo

334 classification. Important, the Kudo's classification differentiates neoplastic and non-

335 neoplastic colon polyps, according to crypts morphology and its lumen size, employing a

336 Narrow Band Image system (NBI) or chromoendoscopy with magnification. Based on Prieto

337 et al., 2016 [30], the perimeter of the crypt lumens was measured and used for crypt pits 
classification by manual and automated analysis of autofluorescence microscopy images. Results show average perimeters of the distal colon crypt lumens coincident with mucosa benign changes (type I and II of Kudo's pit pattern) at the week $4^{\text {th }}$ post-AOM/DSS-treatment [31]. Kudo's work and other such as Tan et al., 2013 [32] were performed by images manual processing obtained from colon mucosa crypt pits with benign morpho-functional changes. Several analysis based on Kudo's pit pattern classification in turn, have employed visual classifications by experienced endoscopists while just a few added an image processing software [30, 33] that work over chromoendoscopy [34, 35] and narrow band imaging endoscopy (NBI) images $[36,37]$. The free-label method apply in the present work shows acceptable results in about 60 seconds, compared with Takemura's procedure that take several minutes [33]. In contrast, the conventional procedure takes around 70 hours from tissue dissection to image reconstruction and analysis [38]. In addition to recognize the geometry of the crypts, endogenous fluorescent molecules allow to identify physiological and biochemical variations during tumoral disease evolution [39-41]. Thus, the fluorescence spectral features which differentiate normal mucosa and adenoma have been identified and empirically correlated with histological diagnosis. However, few works show changes in early stages as observed in this work. Thus, the animals induced to develop cancer presented an intensity peak (statistically extremely significant) at week $8^{\text {th }}$ post-AOM/DSS treatment. In general week $8^{\text {th }}, 16^{\text {th }}$ and $20^{\text {th }}$ post-AOM/DSS treatment showed higher intensity values compared to the control and CCR early stage in week $4^{\text {th }}$. These results are coincident with previous reports $[11,27,29]$ where an increase in autofluorescence signal is detected in dysplastic respect to normal tissues. Cellular autofluorescence is known to depend on the metabolic state [11], which changes not only through several physiological state but also pathological. According excitation wavelengths used in this work, the increased 
362

363

364 Porphyrins have excitation maxima near 400nm, and minor pick to 450-700 nm. Porphyrins

365 also are localized on mitochondrial electron transport strand, highly developed during

autofluorescence from week 4 of CCR induction could be due to hemoglobin breakdown products such as porphyrin derivatives, which are known to accumulate in tumor cells [28]. tumoral state to meet the metabolic demand [42]. Mitochondrias play critical roles in meeting

367 cellular energy demand, in cell death, and in reactive oxygen species (ROS) and stress 368 signaling [11].

Increasing autofluorescence, also can be attributed to lipofuscin and lipofuscin-like compounds dots, which increase under a variety of pathophysiological conditions [43, 44].

371 Between these, oxidative stress and ageing $[45,46]$, characterized by a progressive unbalance

372 between protein damage and clearance, leading at its turn to an increased protein homeostasis 373 disturbance, with accumulation of oxidized proteins' aggregates and, subsequently, of 374 highly-cross linked materials such as lipofuscin and lipofuscin-like lipopigments, affecting 375 cell viability. These cytoplasmic aggregates of undigested cell materials resulting from 376 phagocytosis and autophagy processes and accumulating as endocytoplasmic vesicles under

377 both physiological and pathological conditions, such neoplastic state [47-49].

378 It is known that collagen networks of extracellular matrix is altered from several pathological 379 states, like tumor development [50, 51]. Exploring harmless analysis techniques, recent 380 studies that utilize SHG microscopy have discriminated alterations in collagen fiber 381 scaffoldings associated with non-neoplastic $[52,53]$ and neoplastic diseases $[9,13,54]$. Such spatial alterations may provide cues to cell migration and invasion during cancer progression

$383[55,56]$. Therefore, in order to evaluate the fibrillar networks 3D organization biological 384 tissues, researchers have developed different metrics to quantify data from SHG images [56], 385 such as fibers orientation, spacing, and thickness $[57,58]$. Thus, using anisotropy, energy 
and aspect relation parameters of collagen fibers organization, in this work, the CRC

387 progression was characterized. Fundamentally, the amount and reorientation of the collagen

388 fibers decrease significantly and notably at week $4^{\text {th }}$, an early stage of colon cell-

389 transformation. These results are coincident with our previous [9] and other works [59-61]

390 that analyzed CRC. However, this is the first time that early changes in collagen network is

391 demonstrated by several and harmless methods, and in a complete colon cancer progression.

392 Both linear and nonlinear microscopies, using different strategies, showed that 3D-

393 representations are extremely useful. With linear techniques, crypts transformations were

394 visualized and modifications in autofluorescence intensity observed. In particular, 3D

395 representations using nonlinear techniques allowed to clearly visualizing the modification

396 and change of direction of the collagen fibers around the crypts. We believe that new

397 algorithms are needed to extract quantitative parameters for these stacks.

398 In conclusion, linear and nonlinear signals improve the detection and classification of

399 pathological changes in crypt morphology/distribution and collagen fiber

400 structure/arrangement, and provide a more comprehensive understanding of the connection

401 between $\mathrm{CRC}$ and tissue heterogeneity. In brief, crypt diameter higher than $0.08 \mathrm{~mm}$ and

402 increased fluorescence signal intensity in linear images; and aspect relation above 0.7 and

403 high-energy values obtained from SHG images indicate altered organization, disparate from

404 that characteristic of the normal tissue. The independence of contrast agents arguably

405 improve safety, cost, and time efficiency for diagnosis. In our opinion, technologies that

406 identify reliable changes at the cellular level, characterizing the premalignant state, enable

407 targeted biopsies and have the potential to dramatically reduce the number and cost of

408 random biopsies. In combination with standard screening approaches for CRC, the proposed 
409

410

411

412

413

414

415

416

417

418

419

420

421

422

423

424

425

426

427

428

429

430

methods improve the detection of the disease in its early stages, thereby increasing the chances of successful treatment.

\section{ACKNOWLEDGEMENTS}

J.A. is thankful to IBB-CONICET-UNER for funding (PIO Res: 4337/15; Contract grant number: N8 14620140100004 CO). H.F.C. is thankful to FAPESP for funding $(2009 / 16150-$ 6). INFABiC is co-funded by FAPESP (2014/50938-8) and CNPq (465699/2014-6).

\section{SUPLEMTARY FIGURE LEGENDS}

Figure S1: Pictures of the microscopy systems used. A) The linear analysis was done using an epi-fluorescence microscopy Olympus BX50, adapted to perform optical sectioning and deconvolution methods. B) Nonlinear technique was based in the laser confocal Zeiss LSM780 and a pulsed Ti:Sapphire laser (Spectra-Physics. Irvine, USA) and OPO (APE, Levante, Berlin, Germany) system to perform CARS.

Figure S2: Histopathological CRC progression in mouse AOM/DSS model. S0) (a.1 and a.2): Longitudinal section of normal colon, the Lieberkühn crypts are observed as individual unbranched structures, with normal goblet cell content. S4) (b.1) Image of a pair of crypts with mild dysplasia are observed (arrows). (b.2 and b.3): Injuries known as "aberrant crypt foci" (arrows), in which, elongated, branched and fused crypts are observed. The aberrant crypt cells show hyperchromatic enlarged nuclei, with polarity loss. Also, can be observed a decrease in the goblet cell number. S8) (c.1-c.4): Microadenoma with adenocarcinoma in situ and abundant lymphocytic infiltrates. S16) (d.1): Severe dysplasia (arrow). (d.2-d.4): 
431 Intramucosal adenocarcinoma (arrows). S20) (e.1-e.4): Intramucosal adenocarcinoma, with

432 abundant areas of necrotic debris (arrows).

433 Figure S3: H\&E stain (Left column) and specific stain to detect porphyrin (Midle column)

434 and lipofuscin (Right column) from representative images of CRC model.

435 Figure S4: Automatic quantification of autofluorescence images. Representative images at

436 control, week $4^{\text {th }}, 8^{\text {th }}, 16^{\text {th }}$ and $20^{\text {th }}$ in A) untreated animals (saline injected) and B) AOM

437 injected and DSS treated animals. Below each image the average perimeters of crypts are

438 shown. These quantification and those made in H\&E images are compared and presented in

439 the table, where according to the morphology and perimeter crypts value are classified

440 according Kudo’s criterion.

441 Figure S5: Representative mosaic images during the progression of CRC of a complete colon

442 section showing morphological colon changes (TPEF-green) and collagen arrangement

443 (SHG-red).

444 Figure S6: Collagen arrangement through the CRC progression in: A) submucosa colon

445 regions. B) Quantification of collagen content in mucosa (images not show) and submucosa

446 regions. For calculations 16 ROI (256 x 256 pixels) in each image were selected. Each bar

447 represents the mean $\pm \mathrm{SD}$ of three independent images of each point. Scale bar $=10[\mu \mathrm{m}]$.

448 REFERENCES

449 [1] Siegel R, DeSantis C, Jemal A. Colorectal cancer statistics, 2014. CA Cancer J Clin. $450 \quad 2014 ; 64: 104-117$.

451 [2] Goetz M, Kiesslich R. Advances of endomicroscopy for gastrointestinal physiology and 452 diseases. Am J Physiol Gastrointest Liver Physiol. 2010; 298:G797-G806. 
453 [3] Rosenberg DW, Giardina C, Tanaka T. Mouse models for the study of colon

454 carcinogenesis. Carcinogenesis 2009; 30:183-196.

455 [4] Derry MM, Raina K, Agarwal R, Agarwal C. Characterization of azoxymethane-induced

456 colon tumor metastasis to lung in a mouse model relevant to human sporadic colorectal

457 cancer and evaluation of grape seed extract efficacy. Exp Toxicol Pathol. 2014; 66:235-242.

458 [5] Leslie A, Carey FA, Pratt NR, Steele RJ. The colorectal adenoma-carcinoma sequence.

459 Br J Surg. 2002; 89:845-860.

460 [6] Weissleder R, Pittet MJ. Imaging in the era of molecular oncology. Nature 2008; 452:580-

461589.

462 [7] Kiesslich R, Burg J, Vieth M, Gnaendiger J, Enders M, Delaney P, et al. Confocal laser 463 endoscopy for diagnosing intraepithelial neoplasias and colorectal cancer in vivo. 464 Gastroenterology 2004; 127:706-713.

465 [8] Li L, Li H, Chen Z, et al. Layer-resolved colorectal tissues using nonlinear microscopy.

$466 \quad$ Lasers Med Sci 2015; 30:1589-1597.

467 [9] Bianchi M, Adur J, Ruff SY, Izaguirre MF, Carvalho HF, Cesar CL, et al. Mouse 468 colorectal cancer an early detection approach using nonlinear microscopy. Biomed Mater $469 \quad$ Eng. 2014; 24:3419-3426.

470 [10] Li LH, Chen ZF, Wang XF, Zhuo SM, Li HS, Jiang WZ, et al. Multiphoton microscopy 471 for tumor regression grading after neoadjuvant treatment for colorectal carcinoma. World J

472 Gastroenterol. 2015; 21:4210-4215.

473 [11] DaCosta RS, Andersson H, Cirocco M, Marcon NE, Wilson BC. Autofluorescence 474 characterisation of isolated whole crypts and primary cultured human epithelial cells from 475 normal, hyperplastic, and adenomatous colonic mucosa. J Clin Pathol. 2005; 58:766-774. 
476 [12] Saldua MA, Olsovsky CA, Callaway ES, Chapkin RS, Maitland KC. Imaging

477 inflammation in mouse colon using a rapid stage-scanning confocal fluorescence

478 microscope. J Biomed Opt 2012; 17:016006.

479 [13] Adur J, Carvalho HF, Cesar CL, Casco VH. Nonlinear Microscopy Techniques:

480 Principles and Biomedical Applications. Chapter 6 (Pag 121-149): in Microscopy and

481 Microanalysis 2016; Stefan Stanciu (Ed). Intech. ISBN: 978-953-51-4723-7.

482 [14] Tanaka T. Colorectal carcinogenesis: Review of human and experimental animal

483 studies. J Carcinog. 2009; 8:1-19.

484 [15] Adur J, Diaz-Zamboni J, Vicente N, Izaguirre MF, Casco VH. Digital Deconvolution

485 Microscopy: Development, Evaluation and Utilization in 3D quantitative studies of E-

486 cadherin expression in skin of Bufo arenarun embryos. in Modern Research and Educational

487 Topics in Microscopy 2007; A Mendez-Vilas and J. Diaz (Eds.) Vol II. ISBN-13: 978-84-

$488 \quad 611-9420-9$.

489 [16] Schneider CA, Rasband WS, Eliceiri KW. NIH Image to ImageJ: 25 years of image 490 analysis. Nat Methods. 2012; 9:671-675.

491 [17] Adur J, Pelegati VB, de Thomaz AA, Baratti MO, Andrade LA, Carvalho HF, et al. 492 Second harmonic generation microscopy as a powerful diagnostic imaging modality for 493 human ovarian cancer. J Biophotonics. 2014; 7:37-48.

494 [18] Orchard G, Nation B. Histopathology 2012. Oxford University press.

495 [19] Kudo S, Tamura S, Nakajima T, Yamano H, Kusaka H, Watanabe H. Diagnosis of 496 colorectal tumorous lesions by magnifying endoscopy. Gastrointest Endosc. 1996; 44:8-14.

497 [20] Matteini P, Ratto F, Rossi F, Cicchi R, Stringari C, Kapsokalyvas D, et al. 498 Photothermally induced disordered patterns of corneal collagen revealed by SHG imaging. Opt Express. 2009; 17:4868-4878. 
500 [21] Adur J, DSouza-Li L, Pedroni MV, Steiner CE, Pelegati VB, de Thomaz AA, et al. The

501 severity of Osteogenesis imperfecta and type I collagen pattern in human skin as determined

502 by nonlinear microscopy: proof of principle of a diagnostic method. PLoS One 2013;

$503 \quad 8: e 69186$.

504 [22] Walker RF, Jackway PT, Longstaff ID. Int. J. Pattern. Recogn 2003; 17:17-39.

505 [23] Hurlstone DP, Brown S. Techniques for targeting screening in ulcerative colitis. 506 Postgrad Med J 2007; 83:451-460.

507 [24] Eng J, Lynch RM, Balaban RS. Nicotinamide adenine dinucleotide fluorescence 508 spectroscopy and imaging of isolated cardiac myocytes. Biophys J 1989; 55:621-630.

509 [25] Chance B, Schoener B, Oshino R, Itshak F, Nakase Y. Oxidation-reduction ratio studies 510 of mitochondria in freeze-trapped samples. NADH and flavoprotein fluorescence signals. J

511 Biol Chem. 1979; 254:4764-4771.

512 [26] Bottiroli G, Croce AC, Locatelli D, Marchesini R, Pignoli E, Tomatis S, et al. Natural

513 fluorescence of normal and neoplastic human colon: A comprehensive ex vivo study. Lasers

514 Surg Med. 1995; 16:48-60.

515 [27] Zonios GI, Cothren RM, Arendt JT, Wu J, Van Dam J, Crawford JM, et al. 516 Morphological model of human colon tissue fluorescence. IEEE Trans Biomed Eng 1996; $517 \quad 43: 113-122$.

518 [28] Harris DM, Werkhaven J. Endogenous porphyrin fluorescence in tumors. Lasers Surg 519 Med. 1987; 7:467-472.

520 [29] Wang HW, Willis J, Canto MI, Sivak MV Jr, Izatt JA. Quantitative laser scanning 521 confocal autofluorescence microscopy of normal, premalignant, and malignant colonic 522 tissues. IEEE Trans Biomed Eng. 1999; 46:1246-1252. 
523 [30] Prieto SP, Lai KK, Laryea JA, Mizell JS, Muldoon TJ. Quantitative analysis of ex vivo

524 colorectal epithelium using an automated feature extraction algorithm for microendoscopy

525 image data. J Med Imaging. 2016; 3:024502.

526 [31] Kiesslich R, Galle PR, Neurath MF. Atlas of Endomicroscopy. Springer 2008.

527 [32] Tan CW, Hirokawa Y, Gardiner BS, Smith DW, Burgess AW. Colon cryptogenesis:

528 asymmetric budding. PLoS One 2013, 8:pe78519.

529 [33] Takemura Y, Yoshida S, Tanaka S, Onji K, Oka S, Tamaki T, et al. Quantitative analysis

530 and development of a computer-aided system for identification of regular pit patterns of

531 colorectal lesions. Gastointestinal Endosc 2010; 72:1047-1051.

532 [34] Oliveira dos Santos CE, Malaman D, dos Santos Carvalho T. Malignancy in Large

533 Colorectal Lesions; Arq Gastroenterol. 2014; 3:235-239.

534 [35] Hafner M, Gangl A, Wrba F. Comparison of k-NN, SVM, and NN in pit pattern

535 classification of zoom-endoscopic colon images using co-occurrence histograms. ISPA 2007

536 Proc. 5th Int. Symp. Image Signal Process 2007. Anal. 516-521.

537 [36] Thia KT, Kong CS, Ooi C. Narrow Band Imaging and Autofluorescence Imaging for

538 the Detection and Optical Diagnosis of Colorectal Polyps. 2010;19:51-56.

539 [37] Hayashi N, Tanaka S, Kanao H, Oka S, Yoshida S, Chayama K. Relationship between

540 narrow-band imaging magnifying observation and pit pattern diagnosis in colorectal tumors.

541 Digestion 2013; 87:53-58.

542 [38] Liu CY, Dubé PE, Girish N, Reddy AT, Polk DB. Optical reconstruction of murine

543 colorectal mucosa at cellular resolution. Am J Physiol Gastrointest Liver Physiol. 2015;

$544 \quad 30: 721-735$.

545 [39] Alfano RR, Tang GC, Pradhan A, et al. Fluorescence spectra from cancerous and normal 546 human breast and lung tissue. IEEE J. Quantum Electron 1987; 23:1806-1811. 
547 [40] Cothren RM, Sivak MV, Van Dam J, Petras RE, Fitzmaurice M, Crawford JM, et al.

548 Detection of dysplasia at colonoscopy using laser-induced fluorescence: A blinded study.

549 Gastrointest Endosc. 1996; 44:168-176.

550 [41] Kato M, Uedo N, Ishihara R, Kizu T, Chatani R, Inoue T, et al. Analysis of the color 551 patterns of early gastric cancer using an autofluorescence imaging video endoscopy system.

552 Gastric Cancer 2009; 12:219-224.

553 [42] Epstein T, Gatenby RA, Brown JS. The Warburg effect as an adaptation of cancer cells 554 to rapid fluctuations in energy demand. PLoS One 2017; 18:12(9):e0185085.

555 [43] Białas M, Demczuk S, Dyduch G, Drabik G, Chrupek M, Okoń K. Brown bowel 556 syndrome (intestinal lipofuscinosis) - a case report and review of the literature. Pol J Pathol $557 \quad 2013 ; 64(3): 228-231$.

[44] Di Guardo G. Lipofuscin, lipofuscin-like pigments and autofluorescence. Eur J

$559 \quad$ Histochem 2015; 59:2485.

560 [45] Höhn A, Grune T. Lipofuscin: formation, effects and role of macroautophagy. Redox 561 Biol 2013; 1:140-144.

562 [46] Rattan SI, Keeler KD, Buchanan JH, Holliday R. Autofluorescence as an index of ageing 563 in human fibroblasts in culture. Biosci Rep 1982; 2:561-567.

564 [47] Daum O, Sima R, Mukensnabl P, Vanecek T, Brouckova M, Benes Z, et al. Pigmented 565 solid-pseudopapillary neoplasm of the pancreas. Pathol Int. 2005; 55:280-284.

566 [48] Penouil MH, Gourhant JY, Segretin C, Weedon D4, Rosendahl C. Non-choroidal yellow 567 melanoma showing positive staining with Sudan Black consistent with the presence of 568 lipofuscin: a case report. Dermatol Pract Concept 2014; 4:45-49. 
569 [49] Donatello S, Hudson L, Cottell DC, Blanco A, Aurrekoetxea I, Shelly MJ, et al. An

570 imbalance in progenitor cell populations reflects tumour progression in breast cancer primary

571 culture models. J Exp Clin Cancer Res 2011; 30:45.

572 [50] Furuya Y, Ogata T. Scanning electron microscopic study of the collagen networks of

573 the normal mucosa, hyperplastic polyps, tubular adenoma, and adenocarcinoma of the human

574 large intestine. Tohoku J Exp Med. 1993; 169:1-19.

575 [51] Fang M, Yuan J, Peng C, Li Y. Collagen as a double-edged sword in tumor progression.

576 Tumour Biol. 2014; 35:2871-2882.

577 [52] Abraham T, Hogg J. Extracellular matrix remodeling of lung alveolar walls in three

578 dimensional space identified using second harmonic generation and multiphoton excitation

579 fluorescence. J Struct Biol. 2010; 171:189-196.

580 [53] Sun TL, Liu Y, Sung MC, Chen HC, Yang CH, Hovhannisyan V, et al. Ex vivo imaging

581 and quantification of liver fibrosis using second-harmonic generation microscopy. J Biomed

582 Opt. 2010; 15:036002-036006.

583 [54] Thrasivoulou C, Virich G, Krenacs T, Korom I, Becker DL. Optical delineation of

584 human malignant melanoma using second harmonic imaging of collagen. Biomed Opt 585 Express 2011; 2:1282-1295.

586 [55] Barkan D, Green JE, Chambers AF. Extracellular matrix: a gatekeeper in the transition

587 from dormancy to metastatic growth. Eur J Cancer 2010; 46:1181-1188.

588 [56] Abraham T, Kayra D, McManus B, Scott A. Quantitative assessment of forward and

589 backward second harmonic three dimensional images of collagen type I matrix remodeling 590 in a stimulated cellular environment. J Struct Biol 2012; 180:17-25. 
591 [57] Rao RA, Mehta MR, Toussaint KC. Fourier transform-second-harmonic generation

592 imaging of biological tissues. Opt Express 2009; 17:14534-14542.

593 [58] Chen X, Nadiarynkh O, Plotnikov S, Campagnola PJ. Second harmonic generation

594 microscopy for quantitative analysis of collagen fibrillar structure. Nat Protoc. 2012; 7:654-

595669.

596 [59] Birk JW, Tadros M, Moezardalan K, Nadyarnykh O, Forouhar F, Anderson J, et al.

597 Second harmonic generation imaging distinguishes both high-grade dysplasia and cancer

598 from normal colonic mucosa. Dig Dis Sci. 2014; 59:1529-1534.

599 [60] Zhuo S, Yan J, Chen G, Shi H, Zhu X, Lu J, et al. Label-free imaging of basement 600 membranes differentiates normal, precancerous, and cancerous colonic tissues by second-

601 harmonic generation microscopy. PLoS One 2012; 7(6):e38655.

602 [61] Prieto SP, Greening GJ, Lai KK, et al. Quantitative structural markers of colorectal

603 dysplasia in a cross sectional study of ex vivo murine tissue using label-free multiphoton 604 microscopy. 
UV Lamp / CW Laser

舀
省

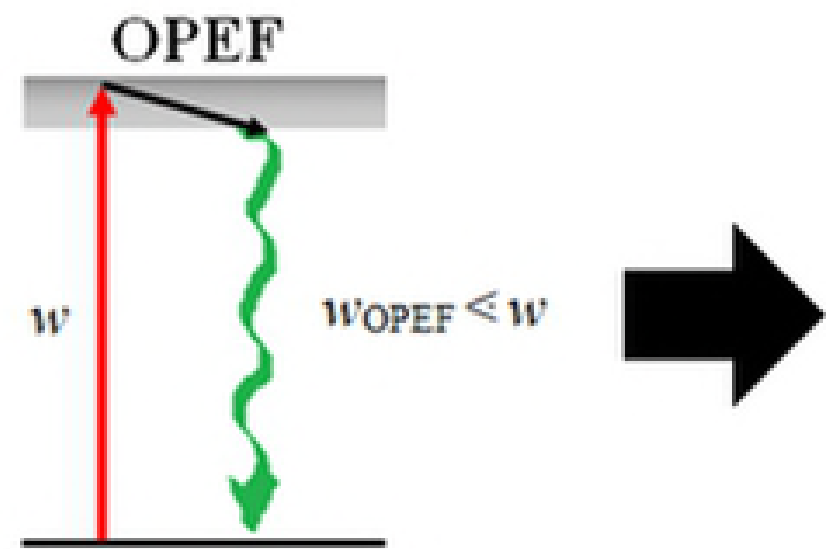

bioRxiv preprint doi: https://doi.org/10.1101/398719; this version posted August 23, 2018. The copyright holder for this preprint (which was not certified by peer review) is the author/funder, who has granted bioRxiv a license to display the preprint in perpetuity. It is made available under aCC-BY 4.0 International license.

$\frac{2}{2}$
$\frac{1}{6}$
$\frac{1}{2}$
$\frac{1}{2}$

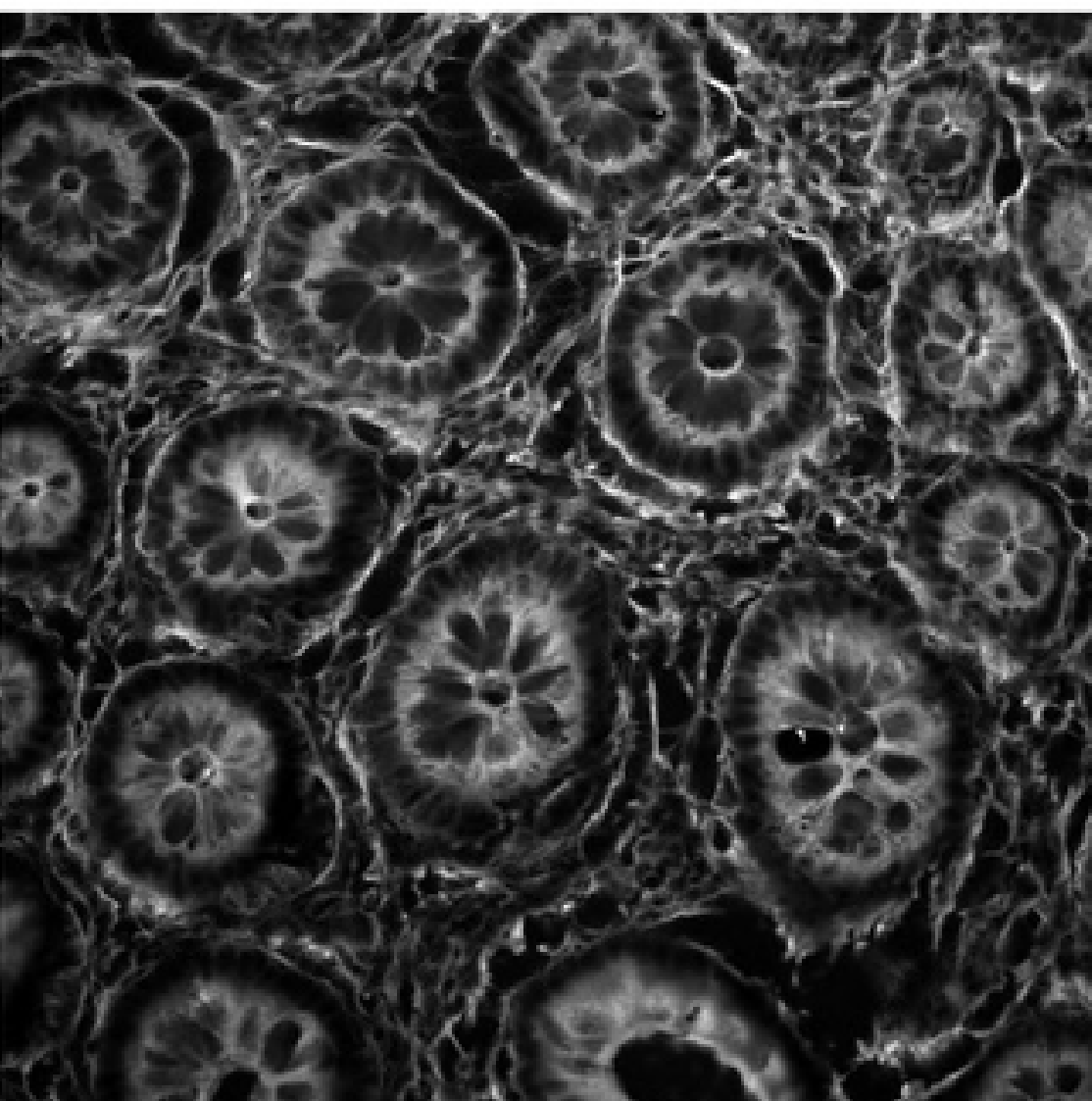

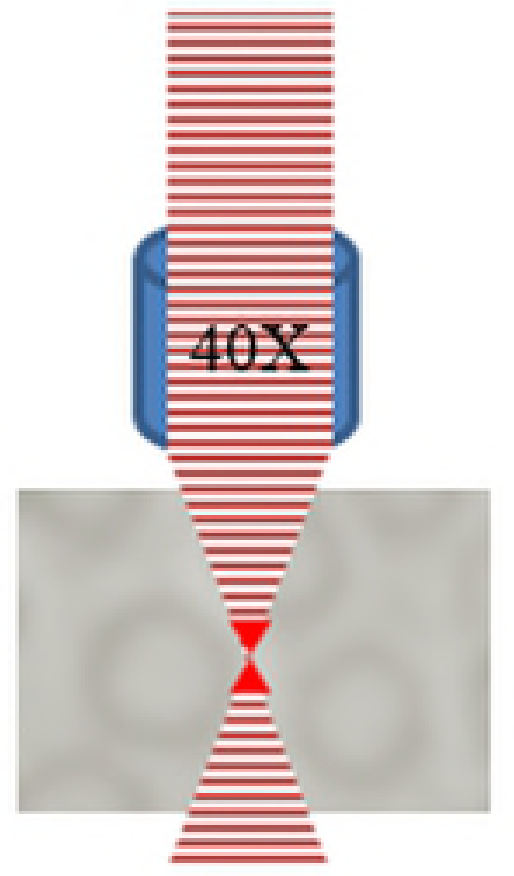

Pulse Laser
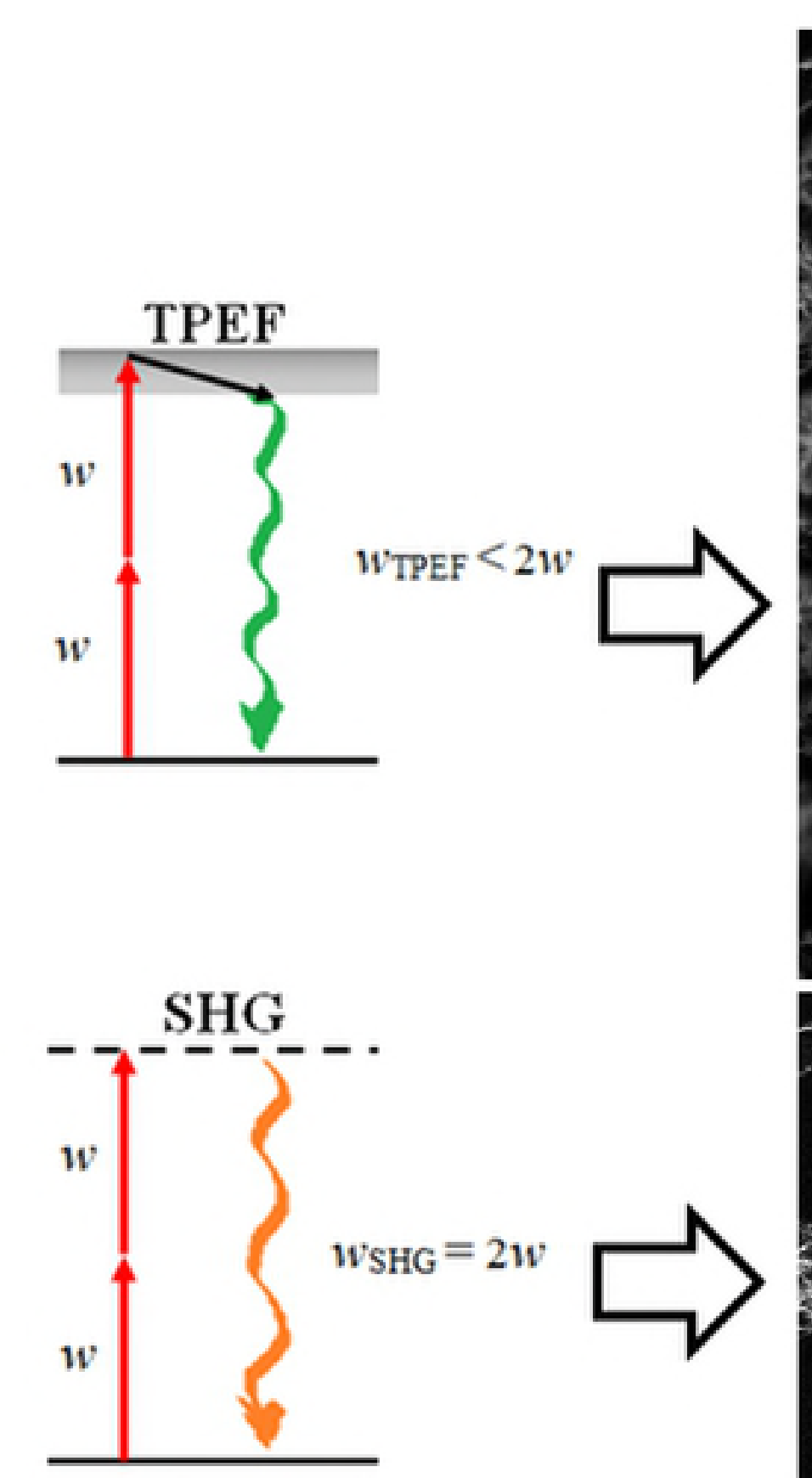

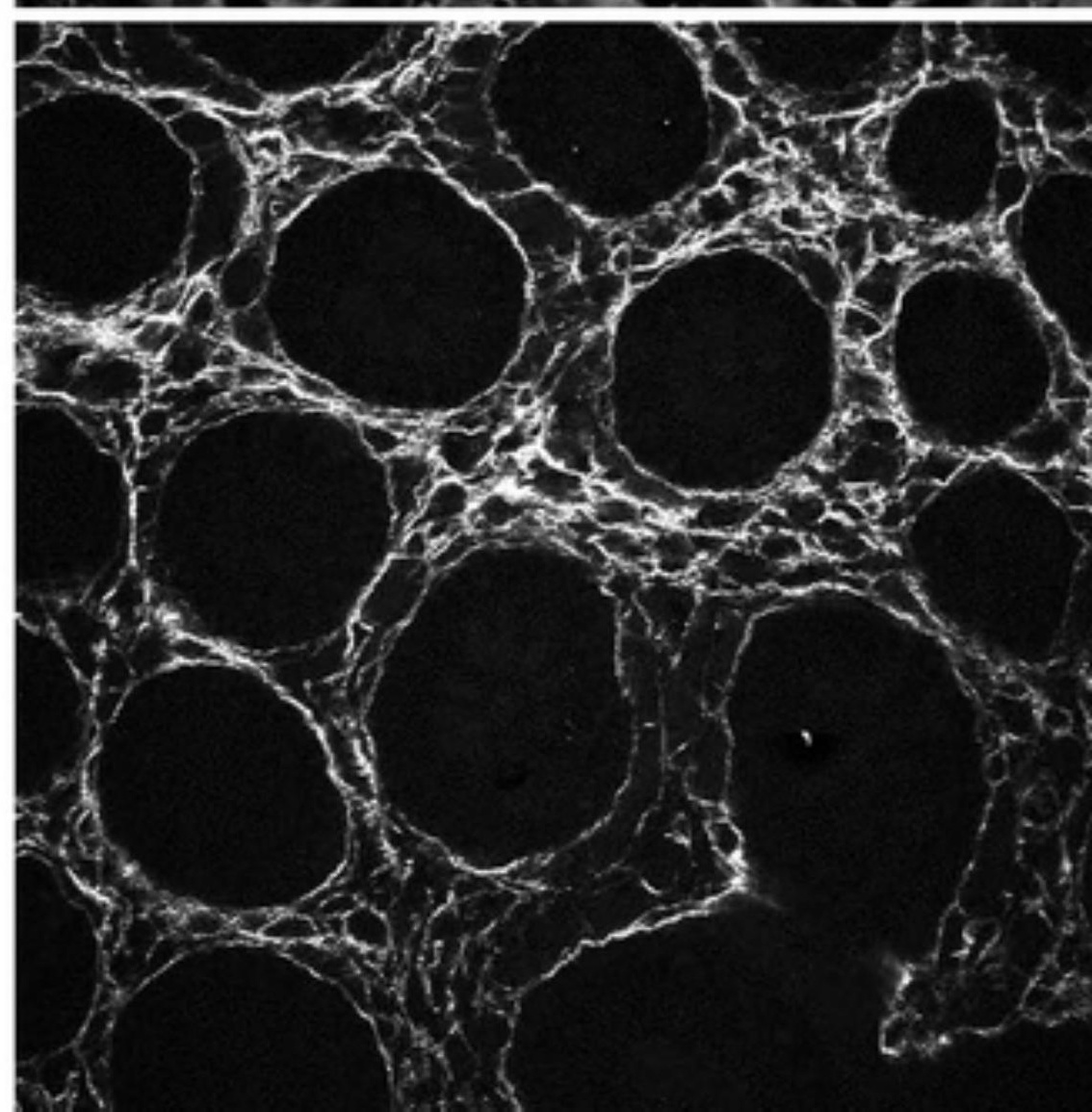



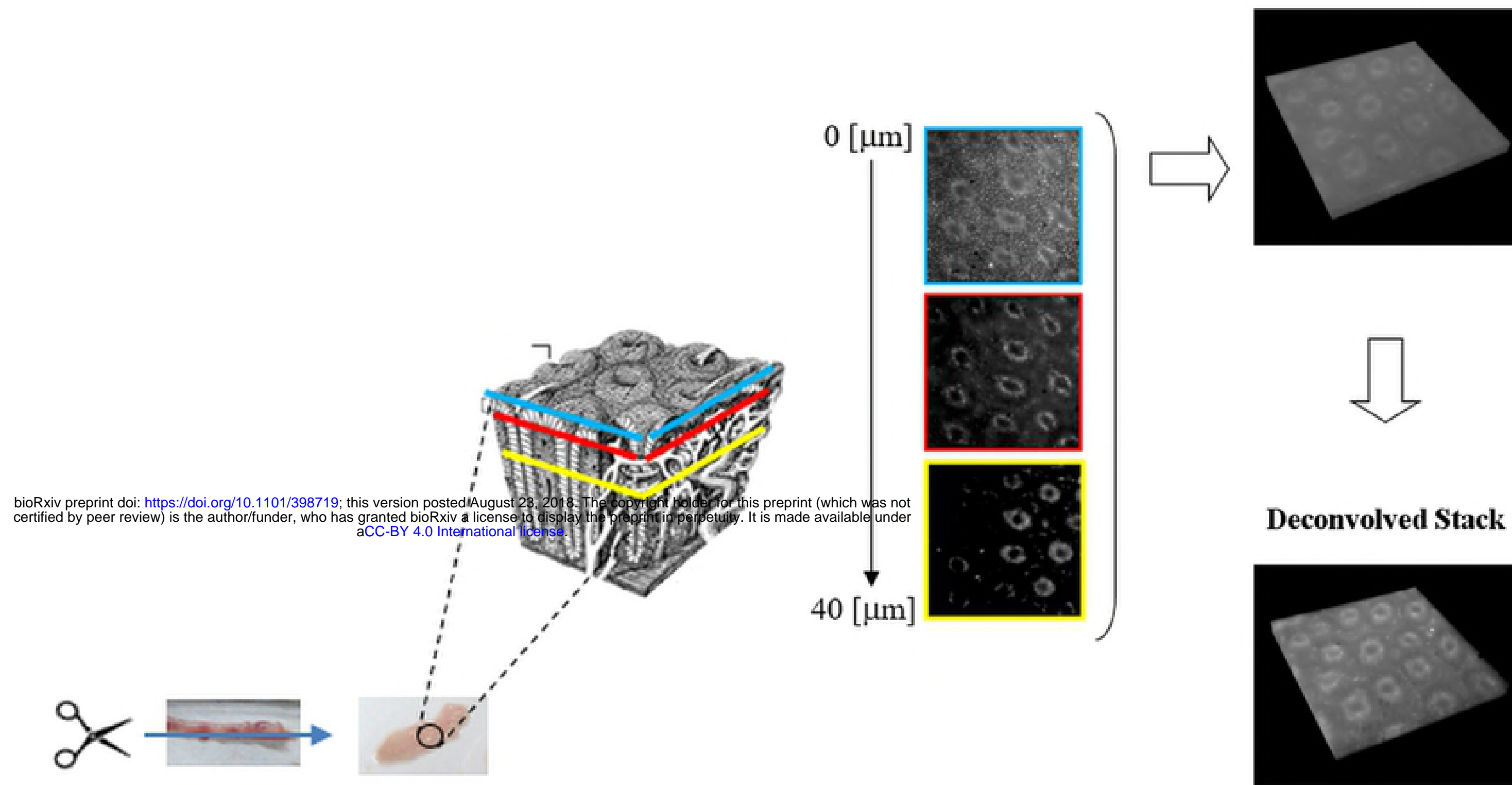

Deconvolved Stack

Longitudinal cut

Transversal cut

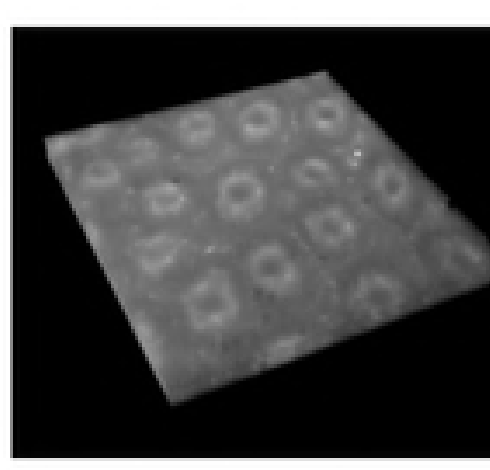

H\&E

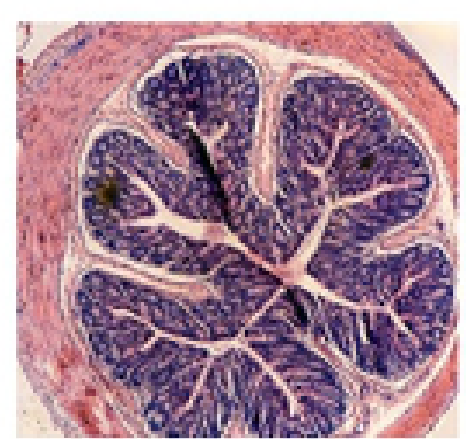

SHG

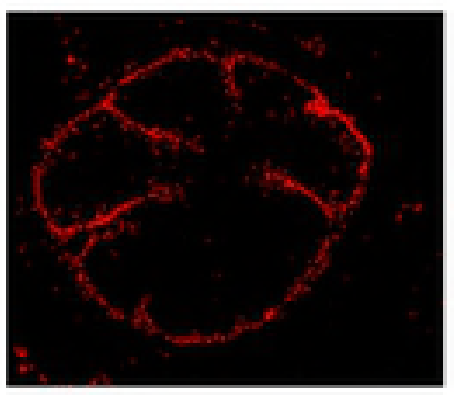

TPEF

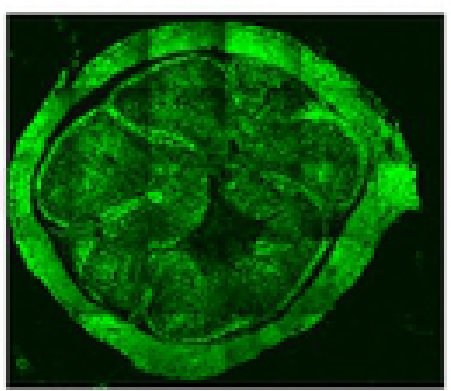

3D Merge

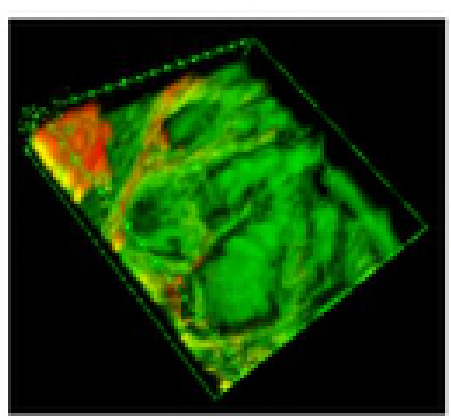

Merge

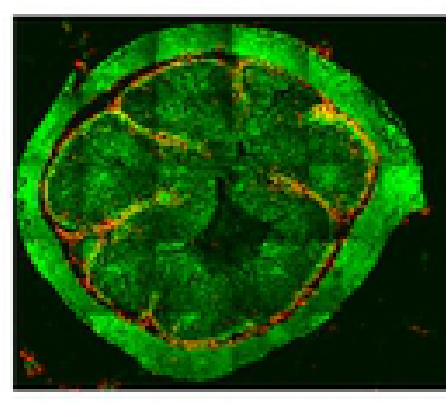


